\title{
BMJ Open RAPID-ADPKD (Retrospective epidemiological study of Asia-Pacific patients with rapId Disease progression of Autosomal Dominant Polycystic Kidney Disease): study protocol for a multinational, retrospective cohort study
}

\author{
Hyunjin Ryu, ${ }^{1}$ Hayne C Park, ${ }^{2}$ Yun Kyu Oh (D) , ${ }^{3}$ Irene Sangadi, ${ }^{4}$ Annette Wong, \\ Changlin Mei, ${ }^{5}$ Tevfik Ecder, ${ }^{6}$ Angela Yee-Moon Wang, ${ }^{7}$ Tze-Wah Kao, ${ }^{8}$ \\ Jenq-Wen Huang, ${ }^{9}$ Gopala K Rangan, ${ }^{4,10}$ Curie Ahn (i) 1,11
}

To cite: Ryu H, Park HC, Oh YK, et al. RAPID-ADPKD (Retrospective epidemiological study of Asia-Pacific patients with rapld Disease progression of Autosomal Dominant Polycystic Kidney Disease): study protocol for a multinational, retrospective cohort study. BMJ Open 2020;10:e034103. doi:10.1136/ bmjopen-2019-034103

- Prepublication history for this paper is available online. To view these files, please visit the journal online (http://dx.doi. org/10.1136/bmjopen-2019034103).

Received 05 September 2019 Revised 09 December 2019 Accepted 20 December 2019

Check for updates

(c) Author(s) (or their employer(s)) 2020. Re-use permitted under CC BY-NC. No commercial re-use. See rights and permissions. Published by BMJ.

For numbered affiliations see end of article.

Correspondence to

Dr Curie Ahn; curie@snu.ac.kr

\section{ABSTRACT}

Introduction Patients with autosomal dominant polycystic kidney disease (ADPKD) reach end-stage renal disease in their fifth decade on average. For effective treatment and early intervention, identifying subgroups with rapid disease progression is important in ADPKD. However, there are no epidemiological data on the clinical manifestations and disease progression of patients with ADPKD from the AsiaPacific region.

Methods and analysis The RAPID-ADPKD (Retrospective epidemiological study of Asia-Pacific patients with rap/d Disease progression of Autosomal Dominant Polycystic Kidney Disease) study is a multinational, retrospective, observational cohort study of patients with ADPKD in the Asia-Pacific region (Australia, China, Hong Kong, South Korea, Taipei and Turkey). This study was designed to identify the clinical characteristics of patients with ADPKD with rapid disease progression. Adult patients with ADPKD diagnosed according to the unified ultrasound criteria and with an estimated glomerular filtration rate $(\mathrm{eGFR}) \geq 45 \mathrm{~mL} / \mathrm{min} / 1.73 \mathrm{~m}^{2}$ at baseline will be included. The cohort will include patients with $\geq 2$ records of eGFR and at least 24 months of follow-up data. Demographic information, clinical characteristics, comorbidities, medications, eGFR, radiological findings that allow calculation of height-adjusted total kidney volume, ADPKD-related complications and the Predicting Renal Outcomes in autosomal dominant Polycystic Kidney Disease (PR0-PKD) score will be collected. Rapid progression will be defined based on the European Renal Association - European Dialysis and Transplant Association (ERA-EDTA) guideline. All other patients without any of these criteria will be classified to be of slow progression. Clinical characteristics will be compared between patients with rapid progression and those with slow progression. The incidence of complications and the effects of race and water intake on renal progression will also be analysed. The planned

\section{Strengths and limitations of this study}

The strength of this study is that this is the first collaboration study in the Asia-Pacific region and will include more than 1000 patients to identify the clinical characteristics of patients with autosomal dominant polycystic kidney disease (ADPKD) with rapid progression.

- Detailed clinical follow-up data of at least 2 years will be collected, including clinical manifestations, laboratory results, renal radiological findings and Predicting Renal Outcomes in autosomal dominant Polycystic Kidney Disease (PRO-PKD) scores, and are expected to provide knowledge on the incidence of renal and extrarenal complications, diagnostic pattern, and the effects of ethnicity and water intake on disease progression.

- Due to the nature of a retrospective observational study, renal function and kidney volume measurement methods could not be unified, and follow-up duration and interval would differ among patients.

- We anticipate more collaboration studies among Asia-Pacific countries will be derived from our study, which can benefit the Asia-Pacific ADPKD population.

sample size of the cohort is 1000 patients, and data from 600 patients have been collected as of 30 May 2019.

Ethics and dissemination This study was approved or is in the process of approval by the institutional review boards at each participating centre. The results will be presented in conferences and published in a journal, presenting data on the clinical characteristics, risk factors for disease progression and patterns of complications of ADPKD in Asian populations. 


\section{INTRODUCTION}

Autosomal dominant polycystic kidney disease (ADPKD) is the most common hereditary renal disease in all races. ${ }^{1}$ About $50 \%$ of patients with ADPKD progress to end-stage renal disease (ESRD) in their fifth decade, and ADPKD is currently the fourth main cause of ESRD. ${ }^{2}$ In addition to renal progression, various renal and extrarenal symptoms and complications occur in patients with ADPKD during their life. ADPKD is a heterogeneous set of diseases caused by a mutation in either two genes, PKD1 and PKD2. Patients with $P K D 2$ mutation show a milder clinical course compared with those with PKD1 mutation. ${ }^{3}$ However, even in the same family, with the same germline mutation in $P K D 1 / P K D 2$ gene, manifestations and severity of disease differ from person to person. ${ }^{2}$ These differences in disease manifestations and progression might be due to various environmental factors, in addition to genetic factors. ${ }^{4}$ Therefore, identifying patients who will develop severe disease is important in the clinical management of
ADPKD. Moreover, since the approval of vasopressin V2 receptor antagonists by the US Food and Drug Administration as the first medication to slow ADPKD progression, identifying the 'rapid progressor' has been a crucial step in deciding which patients will benefit most from the treatment. $^{5}$

However, there have been only a few studies that characterised the nature of the disease and that analysed the progression of ADPKD in the Asia-Pacific population (table 1). A few unique characteristics of the Asian ADPKD population have been noted in previous studies. About $50 \%-80 \%$ of genetic mutations in the PKD1 or PKD2 gene of the Asian population were novel compared with a previous genetic mutation database based on the Western population. $^{6-8}$ In addition, differences in genetic background, race, climate, culture and lifestyle of the Asian population can also affect disease progression differently. Limited data suggest the possibility of faster renal progression in Asian patients with chronic kidney disease (CKD)

\begin{tabular}{|c|c|c|c|c|c|}
\hline Country & Subject of the study & Patients (n) & Study type & $\begin{array}{l}\text { Year of } \\
\text { publication }\end{array}$ & Reference \\
\hline China & Clinical manifestation & 205 & Cross-sectional study & 1995 & 13 \\
\hline China & $\begin{array}{l}\text { Clinical characteristics and } \\
\text { disease progression }\end{array}$ & 541 & $\begin{array}{l}\text { Prospective cohort } \\
\text { study }\end{array}$ & 2014 & 14 \\
\hline China & $\begin{array}{l}\text { Clinical features of inpatients with } \\
\text { ADPKD }\end{array}$ & 168 & Retrospective study & 2018 & 16 \\
\hline Iraq & Clinical manifestation & 30 & Cross-sectional study & 2011 & 17 \\
\hline Japan & Renal progression & 255 & Retrospective study & 2012 & 18 \\
\hline Japan & $\begin{array}{l}\text { Clinical characteristics according } \\
\text { to Mayo classification }\end{array}$ & 296 & Retrospective study & 2019 & 21 \\
\hline Pakistan & Clinical presentation & 56 & Cross-sectional study & 2008 & 22 \\
\hline South Korea & Clinical characteristics & 461 & Cross-sectional study & 2015 & 23 \\
\hline South Korea & Clinical characteristics & 364 & $\begin{array}{l}\text { Prospective cohort } \\
\text { study }\end{array}$ & 2018 & 24 \\
\hline
\end{tabular}

ADPKD, autosomal dominant polycystic kidney disease. 
compared with the Western population. ${ }^{9-11}$ However, previous studies on Asian ADPKD populations were conducted in a single country, which under-represented the whole Asian ADPKD population. ${ }^{12-27}$ Moreover, to date, studies that identified patients with ADPKD with rapid progression were mostly conducted in the Western population. ${ }^{52-30}$ Therefore, a larger, multicentre study is needed to determine the clinical characteristics and to define the risk factors for rapid progression of ADPKD in Asian patients.

The aim of this multinational, multicentre, retrospective cohort study was to determine the clinical characteristics of rapidly progressing patients with ADPKD in the Asia-Pacific region using the current recommendation for identification of rapid progression. ${ }^{5}$ Data on clinical characteristics, complications and disease course of patients with ADPKD in the Asia-Pacific region will be collected. An exploratory goal of this project is to determine whether there are racial differences in disease progression, contributing to a baseline knowledge to inform future studies on this group of patients.

\section{METHODS AND ANALYSIS \\ Study design}

The RAPID-ADPKD (Retrospective epidemiological study of Asia-Pacific patients with rapId Disease progression of Autosomal Dominant Polycystic Kidney Disease) study is a multinational, retrospective observational cohort study designed to determine the prevalence of patients with ADPKD with rapid progression in the Asia-Pacific region based on changes in renal function, kidney size and progression to ESRD.

\section{Organisation}

This study will enrol patients with ADPKD who have visited one of the six hospitals from the six countries in the Asia-Pacific region: (1) Westmead Hospital, Westmead, Australia; (2) Changzheng Hospital, Shanghai, China; (3) Queen Mary Hospital, Hong Kong; (4) Seoul National University Hospital, South Korea; (5) National Taiwan University Hospital, Taipei, Taiwan; and (6) Istanbul Bilim University, Turkey.

\section{Study population}

After retrospectively reviewing patients' data from each hospital, we will enrol adult patients with ADPKD aged $\geq 18$ years old ( $\geq 19$ years old for patients in Taiwan due to the different age criteria for adults in this country) with an estimated glomerular filtration rate (eGFR) $\geq 45 \mathrm{~mL} /$ $\min / 1.73 \mathrm{~m}^{2}$ at the index date of the study. To ensure sufficient follow-up duration for each patient in both the retrospective analysis and in the possible next step of the study, we planned to enrol patients with ADPKD with relatively preserved renal function. The index date is the first available date of reviewed medical records between January 2010 and the enrolment period. Enrolled patients are patients diagnosed with ADPKD according to the unified ultrasound criteria for patients with a family history of $\mathrm{ADPKD}^{31}$ : at least two (unilateral or bilateral) renal cysts at age 18-29, at least two cysts in each kidney at age $30-59$ years, and more than four bilateral renal cysts at age $\geq 60$ years. For patients without a family history of ADPKD, those with clinical diagnosis of ADPKD based on typical radiological findings and/or clinical evaluation will also be included. Patients should have at least two clinical visits with eGFR measurements between 1 January 2010 and at the time at enrolment. In addition, at the time of enrolment, patients should have followed up with the centre during the last 24 months. Patients should also have at least 2 years of clinical follow-up records from the index date (figure 1). Patients with severe heart failure (with symptoms of New York Heart Association class 3 and 4), severe liver disease (Child-Pugh class B or C), chronic inflammatory disease, diabetic nephropathy, vascular disease and/or other comorbidities that can affect renal function will be excluded based on the clinician's judgement. Patients with active cancer who underwent chemotherapy during the observation period will also be excluded from the study. Any medical or surgical conditions that can affect renal function or kidney volume will be excluded as well. The expected sample size of this multinational, retrospective observational cohort is more than 1000 patients.

\section{Data collection}

Demographic information at index date, such as gender, date of birth, race, height and bodyweight, will be collected. Comorbid conditions including diabetes mellitus, dyslipidaemia, hypertension, valvular heart disease, pericardial effusion or left ventricular hypertrophy based on echocardiography, chronic pulmonary disease, coronary artery disease, cerebrovascular disease, and cancer will be investigated. In addition, blood pressure, smoking history and CKD stage based on the Chronic Kidney Disease Epidemiology Collaboration (CKD-EPI) eGFR at index date will also be collected. CKD-EPI eGFR will be calculated using the following formula: eGFR $=141 \times \mathrm{min}$ $(\operatorname{Scr} \times 0.0113 / \mathrm{k}, 1)^{\alpha} \times \max (\operatorname{Scr} \times 0.0113 / \mathrm{k}, 1)^{-1.209} \times$ $0.993^{\text {Age }} \times 1.018$ (if female) $\times 1.159$ (if black), where Scr is serum creatinine, $\mathrm{k}$ is 0.7 for women and 0.9 for men, ${ }^{\alpha}$ is -0.329 for women and -0.411 for men, min indicates the minimum of $\mathrm{Scr} / \mathrm{k}$ or 1 , and max indicates the maximum of $\mathrm{Scr} / \mathrm{k}$ or $1 .{ }^{32}$ Family history of ESRD, medications (antihypertensive agents including calcium channel blocker, ACE, angiotensin receptor blocker and beta blocker, uric acid-lowering agent, antidiabetic agents including metformin, dipeptidyl peptidase-4 (DPP-4) inhibitor and sodium glucose cotransporter (SGLT-2) inhibitor, lipid-lowering agents including statins, and others) and laboratory results (serum albumin, total bilirubin, cholesterol, high-density lipid, low-density lipid, triglyceride, alanine aminotransferase (ALT), aspartate aminotransferase (AST), sodium, potassium, chloride, blood urea nitrogen, glucose and uric acid) will also be collected. At the index date and during the follow-up 
Follow-up

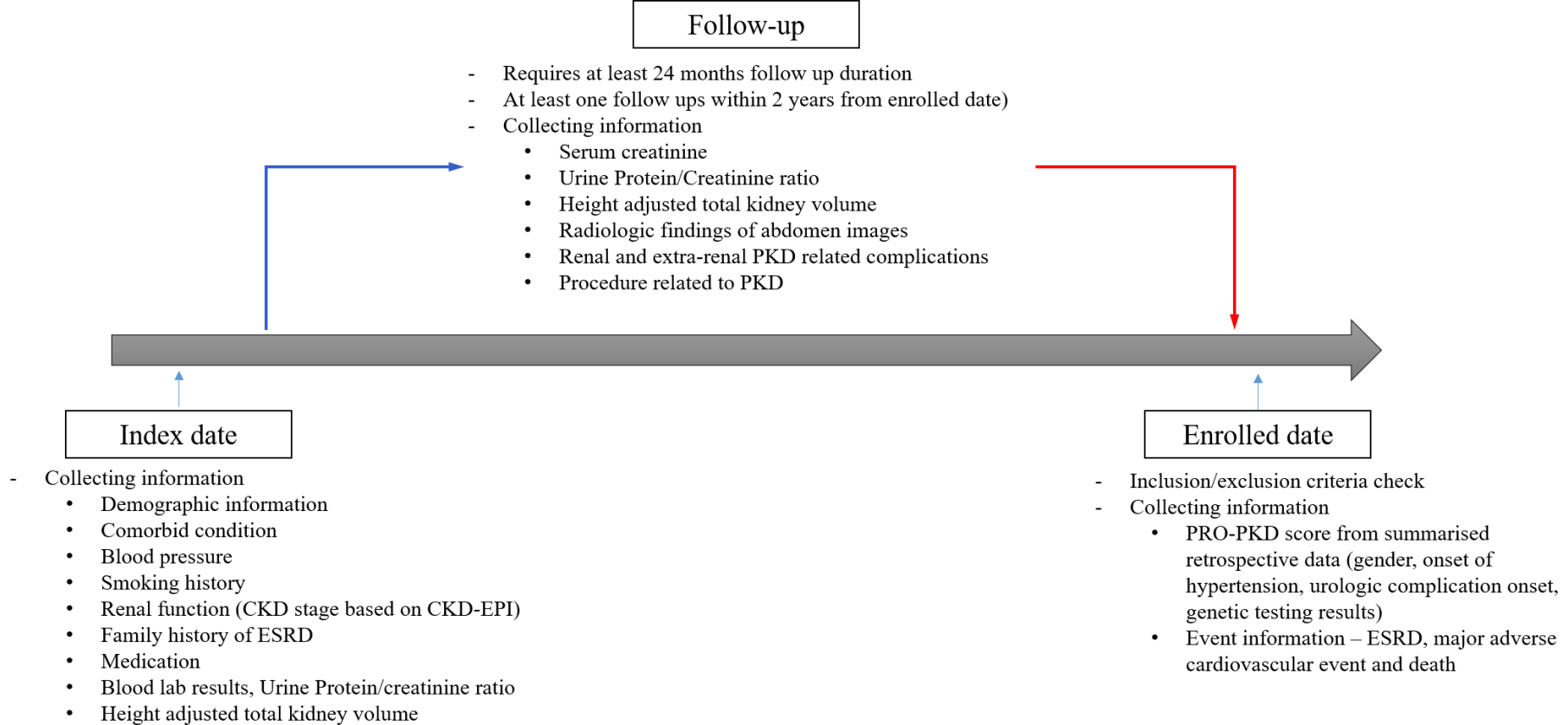

Figure 1 Planned structure and data collection in RAPID-ADPKD study. CKD, chronic kidney disease; CKD-EPI, Chronic Kidney Disease Epidemiology Collaboration; ESRD, end-stage renal disease; PKD, polycystic kidney disease; PRO-PKD, Predicting Renal Outcomes in Autosomal Dominant Polycystic Kidney Disease; RAPID-ADPKD, Retrospective epidemiological study of Asia-Pacific patients with rap/d Disease progression of Autosomal Dominant Polycystic Kidney Disease.

visits, serum creatinine, urine protein:creatinine ratio, height-adjusted total kidney volume (htTKV) from radiological findings on MRI, CT or ultrasound sonography, renal and extrarenal ADPKD-related complications (cyst infection, events or imaging-diagnosed haemorrhagic cysts, proteinuria, kidney stone, gross haematuria, microscopic haematuria, chronic pain, upper urinary tract infection, hypertension, hyperuricaemia, hernia, liver cysts, intracranial aneurysms, subarachnoid haemorrhage, abdominal aorta aneurysms and infertility), and procedures (nephrectomy, sclerotherapy, embolisation, renal replacement therapy, hepatectomy, liver transplantation, fenestration and intervention for intracranial aneurysm) will be investigated.

From the reviewed data, the Predicting Renal Outcomes in autosomal dominant Polycystic Kidney Disease (PROPKD) score and event information including ESRD, major cardiovascular events (myocardial infarction, hospitalisation for unstable angina, transient ischaemic attack and stroke, heart failure requiring hospitalisation and peripheral artery revascularisation procedure) and death will be collected using an electronic case report form. PRO-PKD score will be calculated based on the following: a score of 1 for men, a score of 2 for hypertension before 35 years of age, a score of 2 for having first urological event such as macroscopic haematuria, flank pain or cyst infection before 35 years of age, a score of 2 for non-truncating PKD1 mutation, and a score 4 for truncating PKD1 mutation based on genetic testing results ${ }^{28}$ (figure 1 ).

\section{Serum creatinine measurement and eGFR calculation}

To identify patients with rapid disease progression, standardising creatinine measurement is important. However, this is a multinational retrospective study, and serum creatinine measurement methods inevitably differ from different centres and dates. To reduce error in the interpretation of eGFR due to various creatinine measurement methods used, in addition to collecting the serum creatinine value we will also collect data on creatinine measurement methods such as the Jaffe method (calibrated with isotopic dilution mass spectrometry), enzymatic method, alkaline picrate kinetic, alkaline picrate rate-blanked method with compensation and alkaline picrate rateblanked method without compensation, as supporting data during the analysis. In the calculation of eGFR, if the creatinine measurement was not calibrated with isotopic dilution mass spectrometry, eGFR will be calculated using the $5 \%$ reduced value of the recorded serum creatinine. ${ }^{33}$ All eGFR values will be calculated based on the CKD-EPI equation. $^{32}$

\section{Total kidney volume calculation}

To collect the maximum amount of htTKV data, we will gather data from renal MRI, CT or ultrasound images undertaken during the follow-up. The imaging dates and methods will be collected. If the htTKV has already been measured using imaging, the value and the measurement methods for total kidney volume (TKV) (ellipsoid, stereological measurement or other) will be collected. If there is no measurement done, htTKV will be calculated using the ellipsoid methods. ${ }^{34}$ For the analysis, htTKV calculated by ellipsoid methods would be used mainly to define rapid progression. However, if other methods for calculating htTKV, such as stereological or planimetry, are used on the same patient, the progression will also be determined using the values derived from these methods. 
In both cross-sectional and longitudinal analyses, htTKV data will be analysed and presented according to TKV measurement methods separately and combined.

\section{Follow-up}

Follow-up duration will be defined from the index date until the enrolled date. Date of events such as ESRD, major cardiovascular events and death during the follow-up will be collected and used in the analysis (figure 1).

\section{Outcome variables}

The cohort will include patients with more than two records of eGFR and $\geq 24$ months of follow-up. The primary outcome of the study is the rate of decline in kidney function in the Asia-Pacific ADPKD population. Changes in eGFR slope during the follow-up will be calculated using generalised linear mixed model and compared between patients with rapid progression and those with slow progression. Rapid progression will be defined when any of the following criteria are met, based on European Renal Association - European Dialysis and Transplant Association (ERA-EDTA) recommendations ${ }^{5}$ : (1) an annual eGFR decline $\geq 5 \mathrm{~mL} / \mathrm{min} / 1.73 \mathrm{~m}^{2}$ within 1 year and/or $\geq 2.5 \mathrm{~mL} / \mathrm{min} / 1.73 \mathrm{~m}^{2}$ per year over a period of 5 years; (2) an increase in htTKV $\geq 5 \%$ per year measured from $\geq 3$ radiological images; (3) Mayo classification 1C, 1D or 1E, or kidney length of $>16.5 \mathrm{~cm}$ on ultrasonography; and (4) PKD1 truncated mutation with early symptoms (PRO-PKD score $>6$ ). The remainder of the patients will be classified as slow progressors. The secondary outcome of the study is differences in other clinical characteristics including TKV changes, complication rate, age of complication presentation and treatment aspects between patients with rapid progression and those with slow progression. In addition, a subgroup analysis according to age group and ethnicity will be conducted as secondary outcome measures.

\section{Data collection, monitoring and management}

The entire data collection and monitoring will be managed by a contract research organisation (CRO). The person designated to input data at each centre will be trained by the clinical research associate (CRA) before enrolment of the first patients to ensure uniformity of data at different centres and to minimise data input errors. Patients' eligibility for the study and final inclusion will be decided by the clinician at each centre according to the inclusion and exclusion criteria. All retrospective data will be collected using an electronic case report form. The CRA will visit the centre during the period of data collection and input and will regularly communicate with the person at the centre to determine data input errors and manage possible issues beforehand. Once the data from each centre have been recorded in the case report form, internal monitoring will be conducted. Once the data from all centres have been recorded in the case report form, external monitoring will be performed by the CRO. Missing data on continuous variables will be categorised as 'missing'. Data from 600 patients have been collected as of 30 May 2019.

\section{Statistical methods}

Once all data have been recorded and monitored, statistical analysis will be conducted on both the total study population and each country's population. If a certain variable cannot be collected at a specific centre, the analysis of that variable will be conducted after excluding the missing values. The analysis will be conducted following the guidelines of the International Committee on Harmonisation E9: Statistical Principles for Clinical Trials. $^{35}$

For the primary analysis, all clinical variables will be analysed according to two categories: the rapid progressor and the slow progressor. Additional analysis will be conducted according to CKD stage, age group $(\leq 30,31-40,41-50$ and $\geq 51$ years old), country and race. For the statistical analysis of categorical variables, $\chi^{2}$ test will be used. For continuous variables, Student's t-test and analysis of variance will be conducted. To compare changes in eGFR and htTKV, the generalised linear mixed model will be used to adjust the baseline difference between the individuals. Event data will be analysed using Cox proportional hazard model. $\mathrm{P}<0.05$ will be interpreted as statistically significant. For multiple comparisons, the Holm-Bonferroni method will be used.

\section{Clinical significance of the study}

This RAPID-ADPKD study has been designed to present the clinical data profile of the ADPKD population in the Asia-Pacific region. This study will include patients with ADPKD from six different countries in the Asia-Pacific region-Australia, China, Hong Kong, South Korea, Taiwan and Turkey-and hence will have heterogeneous ADPKD population consisting of different races, such as Han Chinese, Korean, Turks, Kurds, Taiwanese aborigines, Caucasians and so on. Using data from six AsiaPacific countries, we aim to identify the risk factors for the rapid progression of disease in patients with ADPKD in the Asia-Pacific. To address the renal progression of the study population, htTKV and eGFR of the enrolled patients will be retrospectively reviewed and collected.

We also expect to analyse the current status of patients with ADPKD in the Asia-Pacific region, such as the pattern of extrarenal complication, diagnosis, treatment and medical resource utilisation. Patients with ADPKD are known to have a higher risk for hypertension and cardiovascular events compared with the general population. ${ }^{36-38}$ However, in the CKD population, there have been studies reporting that hypertension is better controlled and the incidence of cardiovascular events lower in the Asian population compared with the Western CKD population. ${ }^{39-41}$ Whether the incidence of hypertension and cardiovascular event is relatively low in Asian patients with ADPKD compared with the Western population, as in other patients with CKD, is unknown. In addition, the Asian population has relatively smaller body mass compared with 
the Western population, and there might be a higher incidence of complications due to severe polycystic liver disease and organomegaly in Asian patients with ADPKD. Using the retrospective data from RAPID-ADPKD study, we are planning to analyse the incidence of extrarenal complications in the Asian ADPKD population. Moreover, medical resource utilisation is influenced by the medical system and the government's reimbursement system. Therefore, the diagnostic pattern and assessment methods of disease progression during the follow-up would be different in the Asia-Pacific countries compared with Western countries. For example, MRI is a gold standard tool for TKV measurement, but it is not reimbursed by the medical system in most Asia-Pacific countries; ultrasonography or CT is instead preferred in clinical settings. The results from the current study would provide much information about the current assessment protocol in Asian ADPKD population, which would be valuable in planning for future clinical studies in Asian patients with ADPKD.

In this study, data on ethnicity will also be collected to analyse racial differences in Asian ADPKD population. Only a couple of studies have evaluated ADPKD progression in different races. Freedman et at $t^{42}$ reported that African-American patients with ADPKD showed faster renal progression compared with Caucasian patients with ADPKD in the USA. ${ }^{43}$ With regard to the Asian population, Muto et $a l^{20}$ reported from the Japanese subset study of Tolvaptan Efficacy and Safety in Management of Autosomal Dominant Polycystic Kidney Disease and Its Outcomes (TEMPO) 4:3 trial, that the response to tolvaptan was different in terms of the annual rate of TKV growth in the Japanese population compared with that of the total population. Further studies on racial differences would give us an indepth understanding of the disease pathophysiology and would help identify rapid progressors. Therefore, the effect of race on clinical manifestation and renal progression, which would be analysed in the RAPID-ADPKD study, would be another valuable finding.

It is known that the serum antidiuretic hormone vasopressin level is elevated in dehydrated patients and this is related to the growth of cysts in ADPKD.$^{44}$ Based on this, sufficient water intake, as well as vasopressin V2 receptor antagonist, has been practised to slow renal progression in patients with ADPKD. ${ }^{45}$ While the beneficial effect of increased water intake has been supported by animal studies, there are not enough human studies to support this recommendation. A randomised controlled trial to determine the efficacy and safety of prescribed water intake to prevent kidney failure due to ADPKD (PREVENT-ADPKD), is currently under way. ${ }^{45}$ In the RAPID-ADPKD study, by collecting and analysing data on spot urine specific gravity as a marker of water intake in a relatively large number of patients, we expect to provide baseline data on the effect of water intake in patients with ADPKD.

\section{Limitations}

Since this is a retrospective, observational cohort study from six different countries, we expect several limitations will exist. As mentioned earlier, eGFR and htTKV, the two major indicators of disease progression in ADPKD, cannot be measured or interpreted using a unified method. Additionally, follow-up intervals or htTKV measurements will be different among different participating countries. In addition, there might be an insufficient number of patients who can provide genetic data, which is a known important prognostic marker of renal progression in ADPKD. A minimum follow-up data of 2 years would not be sufficient to identify patients with rapid progression, as suggested in the ERA-EDTA guideline. Also, a population with relatively preserved renal function would not show enough renal changes during a minimum follow-up of 2 years. Moreover, we could not evaluate renal function using cystatin $\mathrm{C}$ or inulin clearance, which would be more appropriate methods especially for patients with sarcopaenia with severe polycystic liver disease. Nevertheless, we believe that our analysis using eGFR and htTKV would provide valuable findings since we will collect data from actual clinical practice and from a large number of patients across different countries. Despite some limitations due to the nature of a multinational, retrospective observational study, we expect that our results will provide valuable knowledge on the clinical characteristics and the rapid progression of Asian patients with ADPKD, and for the first time will provide basic information on current practice in the Asia-Pacific region. Based on the findings of this study, we hope other well-organised multinational prospective studies are designed and conducted to identify the clinical characteristics and unique risk factors of the Asian ADPKD population.

\section{Ethics and dissemination}

This study is a retrospective, observational cohort study, and there are no interventions provided to the study subjects. Therefore, this study presents no more than minimal risk of harm to the study subjects. Also, all institutional review boards approved the consent waiver. All data collected for the study will be managed according to a non-disclosure agreement and privacy legislation. Data will be stored for at least 5 years after completion of enrolment but can be deleted according to each country's privacy regulations. The results will be presented in conferences and published in a journal, presenting data on the clinical characteristics, risk factors for disease progression and patterns of complications of ADPKD in Asian populations.

\section{Patient and public involvement}

In this study, patients or the public were not involved in the design, conduct, reporting or dissemination of research.

Author affiliations

${ }^{1}$ Department of Internal Medicine, Seoul National University Hospital, Seoul, Korea (the Republic of) 
${ }^{2}$ Department of Internal Medicine, Kangnam Sacred Heart Hospital, Seoul, Korea (the Republic of)

${ }^{3}$ Department of Internal Medicine, Seoul National University-Seoul Metropolitan Government Boramae Medical Center, Seoul, Korea (the Republic of)

${ }^{4}$ Department of Renal Medicine, Westmead Hospital, Western Sydney Local Health District, Westmead, New South Wales, Australia

${ }^{5}$ Department of Nephrology, Kidney Institute, Changzheng Hospital, Second Military Medical University, Shanghai, China

${ }^{6}$ Department of Internal Medicine, Istanbul Bilim Universitesi, Istanbul, Turkey ${ }^{7}$ Department of Internal Medicine, Queen Mary Hospital, University of Hong Kong, Hong Kong SAR, China

${ }^{8}$ Department of Internal Medicine, Fu Jen Catholic University Hospital, New Taipei City, Taiwan

${ }^{9}$ Division of Nephrology, National Taiwan University Hospital, Taipei, Taiwan

${ }^{10}$ Centre for Transplant and Renal Research, The Westmead Institute for Medical Research, Sydney, New South Wales, Australia

${ }^{11}$ Department of Internal Medicine, Seoul National University, Seoul, Korea (the Republic of

\section{Twitter Angela Yee-Moon Wang @aymwanghkuhk}

Contributors HR participated in the design of the study, data acquisition, data analysis and drafting of the manuscript. HCP and YKO participated in the design of the study, interpretation of data and revising the manuscript. IS, AW, CM, TE, AY-MW, T-WK and J-WH participated in the design of the study, and in the acquisition and monitoring of data to improve accuracy. GKR participated in the design of the study, acquisition and interpretation of the data, revising the manuscript and in the final approval of the manuscript for publication. CA designed the study, gathered the study collaborators and finalised the manuscript. All authors read and approved the manuscript.

Funding This work was supported by Korea Otsuka International Asia Arab. This funding source had no role in the design of this study and will not have any role during its execution, analyses, data interpretation or decision to submit the results.

Competing interests GKR received grant support from Danone Nutricia Research for clinical research on ADPKD.

Patient consent for publication Not required.

Ethics approval The basic protocol of the study was approved by most of the participating centres' institutional review boards: Seoul National University Hospital, Seoul (no 1801-114-917); Istanbul Bilim University Hospital, Turkey (no 19.12.2017/65-11); National Taiwan University Hospital, Taiwan (no 201801072RSA); Westmead Hospital, Australia (no LNR/17/WMEAD/444); and Shanghai Changzheng Hospital, China (no 2018SL025). Approval is currently in process with Queen Mary Hospital of Hong Kong.

Provenance and peer review Not commissioned; externally peer reviewed.

Open access This is an open access article distributed in accordance with the Creative Commons Attribution Non Commercial (CC BY-NC 4.0) license, which permits others to distribute, remix, adapt, build upon this work non-commercially, and license their derivative works on different terms, provided the original work is properly cited, appropriate credit is given, any changes made indicated, and the use is non-commercial. See: http://creativecommons.org/licenses/by-nc/4.0/.

\section{ORCID iDs}

Yun Kyu Oh http://orcid.org/0000-0001-8632-5743

Curie Ahn http://orcid.org/0000-0001-7033-1102

\section{REFERENCES}

1 Torres VE, Harris PC, Pirson Y. Autosomal dominant polycystic kidney disease. Lancet 2007;369:1287-301.

2 Torres VE, Harris PC. Autosomal dominant polycystic kidney disease: the last 3 years. Kidney Int 2009;76:149-68.

3 Hwang Y-H, Conklin J, Chan W, et al. Refining genotype-phenotype correlation in autosomal dominant polycystic kidney disease. J Am Soc Nephrol 2016;27:1861-8.

4 Chapman AB, Devuyst O, Eckardt K-U, et al. Autosomal-Dominant polycystic kidney disease (ADPKD): Executive summary from a kidney disease: improving global outcomes (KDIGO) controversies conference. Kidney Int 2015;88:17-27.

5 Gansevoort RT, Arici M, Benzing T, et al. Recommendations for the use of tolvaptan in autosomal dominant polycystic kidney disease: a position statement on behalf of the ERA-EDTA working groups on inherited kidney disorders and European renal best practice. Nephro Dial Transplant 2016;31:337-48.

6 Choi R, Park HC, Lee K, et al. Identification of novel PKD1 and Pkd2 mutations in Korean patients with autosomal dominant polycystic kidney disease. BMC Med Genet 2014;15:129.

$7 \mathrm{Xu} \mathrm{D}, \mathrm{Ma}$ Y, Gu X, et al. Novel mutations in the PKD1 and PKD2 genes of Chinese patients with autosomal dominant polycystic kidney disease. Kidney Blood Press Res 2018;43:297-309.

8 Chang M-Y, Chen H-M, Jenq C-C, et al. Novel PKD1 and Pkd2 mutations in Taiwanese patients with autosomal dominant polycystic kidney disease. J Hum Genet 2013;58:720-7.

9 Barbour SJ, Er L, Djurdjev O, et al. Differences in progression of CKD and mortality amongst Caucasian, Oriental Asian and South Asian CKD patients. Nephrol Dial Transplant 2010;25:3663-72.

10 Mathur R, Dreyer G, Yaqoob MM, et al. Ethnic differences in the progression of chronic kidney disease and risk of death in a UK diabetic population: an observational cohort study. BMJ Open 2018;8:e020145.

11 Barbour SJ, Cattran DC, Kim SJ, et al. Individuals of Pacific Asian origin with IgA nephropathy have an increased risk of progression to end-stage renal disease. Kidney Int 2013;84:1017-24.

12 Ravine D, Walker RG, Gibson RN, et al. Phenotype and genotype heterogeneity in autosomal dominant polycystic kidney disease. Lancet 1992;340:1330-3.

13 Liu Y, Zhang $\mathrm{H}$, Zhong $\mathrm{H}$. [Systemic manifestations of adult polycystic kidney disease: an analysis of 205 cases]. Zhonghua Nei Ke Za Zhi 1995;34:612-5.

14 Chen D, Ma Y, Wang X, et al. Clinical characteristics and disease predictors of a large Chinese cohort of patients with autosomal dominant polycystic kidney disease. PLoS One 2014;9:e92232.

15 Jin M, Xie Y, Chen Z, et al. System analysis of gene mutations and clinical phenotype in Chinese patients with autosomal-dominant polycystic kidney disease. Sci Rep 2016;6:35945.

16 Meng J, Xu Y, Li A, et al. Clinical features of 167 inpatients with autosomal dominant polycystic kidney disease at a single center in China. Med Sci Monit 2018;24:6498-505.

17 Alsaedi AJ, Jamal H, Al-Windawi S. The prevalence of hypertension and nephrolithiasis in a sample of Iraqi patients with autosomaldominant polycystic kidney disease. Saudi J Kidney Dis Transpl 2011;22:1044-5.

18 Higashihara E, Horie S, Muto S, et al. Renal disease progression in autosomal dominant polycystic kidney disease. Clin Exp Nephrol 2012;16:622-8.

19 Kurashige M, Hanaoka K, Imamura M, et al. A comprehensive search for mutations in the PKD1 and PKD2 in Japanese subjects with autosomal dominant polycystic kidney disease. Clin Genet 2015;87:266-72

20 Muto S, Kawano H, Higashihara E, et al. The effect of tolvaptan on autosomal dominant polycystic kidney disease patients: a subgroup analysis of the Japanese patient subset from tempo 3:4 trial. Clin Exp Nephrol 2015;19:867-77.

21 Higashihara E, Yamamoto K, Kaname S, et al. Age- and heightadjusted total kidney volume growth rate in autosomal dominant polycystic kidney diseases. Clin Exp Nephrol 2019;23:100-11.

22 Rabbani MA, Ali SS, Murtaza G, et al. Clinical presentation and outcome of autosomal dominant polycystic kidney disease in Pakistan: a single center experience. J Pak Med Assoc 2008;58:305-9.

$23 \mathrm{Kim} \mathrm{H}$, Park HC, Ryu H, et al. Clinical correlates of mass effect in autosomal dominant polycystic kidney disease. PLoS One 2015;10:e0144526.

$24 \mathrm{Kim} \mathrm{H}$, Koh J, Park SK, et al. Baseline characteristics of the autosomal dominant polycystic kidney disease Subcohort of the Korean cohort study for outcomes in patients with chronic kidney disease (KNOW-CKD). Nephrology 2018.

25 Sung $\mathrm{P}-\mathrm{H}$, Chiang $\mathrm{H}-\mathrm{J}$, Yang $\mathrm{Y}-\mathrm{H}$, et al. An association between autosomal-dominant polycystic kidney disease and the risk of acute myocardial infarction in Asian population - results of a nationwide study. Oncotarget 2017;8:19365-75.

26 Kazancioglu R, Ecder T, Altintepe L, et al. Demographic and clinical characteristics of patients with autosomal dominant polycystic kidney disease: a multicenter experience. Nephron Clin Pract 2011;117:c270-5.

27 Ozkok A, Akpinar TS, Tufan F, et al. Clinical characteristics and predictors of progression of chronic kidney disease in autosomal dominant polycystic kidney disease: a single center experience. Clin Exp Nephrol 2013;17:345-51

28 Cornec-Le Gall E, Audrézet M-P, Rousseau A, et al. The PROPKD score: a new algorithm to predict renal survival in autosomal dominant polycystic kidney disease. J Am Soc Nephrol 2016;27:942-51. 
29 Irazabal MV, Rangel LJ, Bergstralh EJ, et al. Imaging classification of autosomal dominant polycystic kidney disease: a simple model for selecting patients for clinical trials. J Am Soc Nephrol 2015;26:160-72.

30 Schrier RW, Brosnahan G, Cadnapaphornchai MA, et al. Predictors of autosomal dominant polycystic kidney disease progression. J Am Soc Nephrol 2014;25:2399-418.

31 Pei Y, Obaji J, Dupuis A, et al. Unified criteria for ultrasonographic diagnosis of ADPKD. J Am Soc Nephrol 2009;20:205-12.

32 Levey AS, Stevens LA, Schmid $\mathrm{CH}$, et al. A new equation to estimate glomerular filtration rate. Ann Intern Med 2009;150:604-12.

33 Skali $\mathrm{H}$, Uno H, Levey AS, et al. Prognostic assessment of estimated glomerular filtration rate by the new chronic kidney disease epidemiology collaboration equation in comparison with the modification of diet in renal disease study equation. Am Heart $J$ 2011;162:548-54.

34 O'Neill WC, Robbin ML, Bae KT, et al. Sonographic assessment of the severity and progression of autosomal dominant polycystic kidney disease: the Consortium of renal imaging studies in polycystic kidney disease (CRISP). Am J Kidney Dis 2005;46:1058-64.

35 Lewis JA. Statistical principles for clinical trials (ICH E9): an introductory note on an international guideline. Stat Med 1999:18:1903-42.

36 Helal I, Reed B, Mettler P, et al. Prevalence of cardiovascular events in patients with autosomal dominant polycystic kidney disease. Am J Nephrol 2012;36:362-70.

37 Massella L, Mekahli D, Paripović D, et al. Prevalence of hypertension in children with early-stage ADPKD. Clin J Am Soc Nephrol 2018;13:874-83.
38 Fick GM, Johnson AM, Hammond WS, et al. Causes of death in autosomal dominant polycystic kidney disease. J Am Soc Nephrol 1995;5:2048-56.

39 Alencar de Pinho N, Levin A, Fukagawa M, et al. Considerable international variation exists in blood pressure control and antihypertensive prescription patterns in chronic kidney disease. Kidney Int 2019;96:983-94.

40 Tanaka K, Watanabe T, Takeuchi A, et al. Cardiovascular events and death in Japanese patients with chronic kidney disease. Kidney Int 2017;91:227-34.

41 Jolly SE, Burrows NR, Chen S-C, et al. Racial and ethnic differences in mortality among individuals with chronic kidney disease: results from the kidney early evaluation program (keep). Clin J Am Soc Nephrol 2011;6:1858-65.

42 Freedman BI, Soucie JM, Chapman A, et al. Racial variation in autosomal dominant polycystic kidney disease. Am J Kidney Dis 2000;35:35-9.

43 Murphy EL, Dai F, Blount KL, et al. Revisiting racial differences in ESRD due to ADPKD in the United States. BMC Nephrol 2019;20:55.

44 Gattone VH, Wang X, Harris PC, et al. Inhibition of renal cystic disease development and progression by a vasopressin $\mathrm{V} 2$ receptor antagonist. Nat Med 2003;9:1323-6.

45 Wong ATY, Mannix C, Grantham JJ, et al. Randomised controlled trial to determine the efficacy and safety of prescribed water intake to prevent kidney failure due to autosomal dominant polycystic kidney disease (PREVENT-ADPKD). BMJ Open 2018;8:e018794. 